\title{
Converting oral to long-acting injectable antipsychotics: a guide for the perplexed-CORRIGENDUM
}

\author{
Jonathan M. Meyer
}

doi.org/10.1017/S1092852917000840. Published by Cambridge University Press, 19 January 2018.

In the original publication of "Converting oral to longacting injectable antipsychotics: a guide for the perplexed," some errors appeared in Tables 1 and 2 and on pages 23-24. The corrections made to rectify these errors are noted below, and the article has been corrected online.

Table 1, second column: the headers "Aripiprazole" and "Dehydroaripiprazole" were shifted to the right; the number "33333333" was deleted

Table 2, fourth column: "84-95 days (deltoid)" was replaced with "30-33"; "118-139 days (gluteal)" was deleted

Table 2, fifth column: "30-33" was replaced with "84-95 days (deltoid)"; "118-139 days (gluteal)" was added Page 23: "Naturalistic data from a cohort of 217 German patients treated with oral paliperidone ER show a dose correlation of $4.7( \pm 2.9) \mathrm{ng} / \mathrm{ml}$ for every milligram of oral paliperidone" was replaced with "Naturalistic data from 217 patients treated with oral paliperidone ER show a dose correlation of $4.7( \pm 2.9) \mathrm{ng} / \mathrm{ml}$ per milligram."
Page 23: "Using this formula, a mean plasma level of $28.4 \mathrm{ng} / \mathrm{ml}$ seen with $234 \mathrm{mg}$ of paliperidone palmitate after 12 weeks appears comparable to approximately $6 \mathrm{mg} / \mathrm{d}$ of oral paliperidone" was changed to "However, this provides dosing guidance 2-fold higher than PK models of larger samples. The package insert thus recommends that paliperidone palmitate 117 month equals $6 \mathrm{mg}$ of oral paliperidone."

Page 24: "however, the half-life is markedly longer than other LAI preparations and this property not only permits" was changed to "however, the long half-life of 53.9-57.2 days not only permits"

The author and publisher apologize for these errors.

\section{REFERENCE:}

Meyer JM. Converting oral to long-acting injectable antipsychotics: a guide for the perplexed. CNS Spectrums. 2017; 22(S1): 18-27. 